\title{
Risk of pulmonary embolism and deep venous thrombosis in patients with asthma: a nationwide case-control study from Sweden
}

\author{
Bengt Zöller, Mirnabi Pirouzifard, Ashfaque A. Memon, Jan Sundquist and \\ Kristina Sundquist \\ Affiliation: Center for Primary Health Care Research, Lund University, Malmö, Sweden. \\ Correspondence: Dr Bengt Zöller, Center for Primary Health Care Research, Clinical Research Center, Floor 11 , \\ Building 28, Entrance 72, Malmö University Hospital, S-205 02 Malmö, Sweden. E-mail: bengt.zollerlamed.lu.se
}

@ERSpublications

Asthma is associated with an increased risk of pulmonary embolism and venous thrombosis http://ow.ly/fk3U306j5Dr

Cite this article as: Zöller B, Pirouzifard M, Memon AA, et al. Risk of pulmonary embolism and deep venous thrombosis in patients with asthma: a nationwide case-control study from Sweden. Eur Respir J 2017; 49: 1601014 [https://doi.org/10.1183/13993003.01014-2016].

ABSTRACT Asthma is associated with an increased risk of pulmonary embolism (PE) but little is known about whether asthma is associated with an increased risk of deep venous thrombosis (DVT). The aim in this study was to determine the risk of the first event of PE, DVT or a combination of PE and DVT in patients with asthma.

We conducted this nationwide case-control study using data from Swedish nationwide registries. We included 114366 Swedish-born patients with a first hospital diagnosis of PE, 76494 patients with DVT and 6854 patients with both PE and DVT in Sweden between 1981 and 2010. We also included five age-, sexand education-matched population controls. All previous hospital diagnoses of asthma were identified. Conditional logistic regression was used to compute odds ratios with adjustment for potential confounders.

Asthma was associated with an adjusted odds ratio for PE of 1.43 (95\% CI 1.37-1.50), for DVT of 1.56 (95\% CI 1.47-1.65) and for combined PE and DVT of 1.60 (95\% CI 1.32-1.93). Asthma was associated with an increased risk of PE, DVT and combined PE and DVT.

Thus, the inflammation conferred by asthma seems to have systemic (and not just local) prothrombotic effects with increased risk of both DVT and PE.

This article has supplementary material available from erj.ersjournals.com

Received: May 202016 | Accepted after revision: Nov 112016

Support statement: This work was supported by grants awarded to B. Zöller by the Swedish Heart-Lung Foundation and Region Skåne; ALF funding awarded to B. Zöller, K. Sundquist and J. Sundquist by Region Skåne; grants awarded to B. Zöller, K. Sundquist and J. Sundquist by the Swedish Research Council; and grants awarded to J. Sundquist by the Swedish Council for Working Life and Social Research and King Gustaf V and Queen Victoria's Foundation of Freemasons. Funding information for this article has been deposited with the Open Funder Registry.

Conflict of interest: None declared.

Copyright OERS 2017 


\section{Introduction}

Chronic inflammatory diseases such as inflammatory bowel disease, rheumatoid arthritis, systemic lupus erythematosus and Behçet's syndrome are associated with the activation of coagulation and an increased risk of venous thromboembolism (VTE) [1-3]. Asthma is a heterogeneous group of conditions characterised by recurrent reversible bronchial obstruction [4]. The disease is usually associated with bronchial hyper-responsiveness and evidence of chronic airway inflammation [4]. Asthma has also been associated with procoagulant and antifibrinolytic activity in the airways [5]. Consequently, two reports have found an association between asthma and an increased risk of VTE [6, 7]. MAJoor et al. studied 648 patients with asthma (283 with severe and 365 with mild-to-moderate asthma) [6]. They found an increased risk for pulmonary embolism (PE) but not deep venous thrombosis (DVT) in asthma patients. The risk was higher in patients with severe asthma (hazard ratio (HR) 8.93, 95\% CI 4.62-15.63), than in patients with mild-to-moderate asthma $(\mathrm{HR}=3.56,95 \% \mathrm{CI} 0.97-9.12)$. The risk was also higher in patients treated with systemic glucocorticoids. In a nationwide study from Taiwan, findings from 31356 asthma patients confirmed an increased risk of PE with a HR of 3.24 [7]. However, the risk of DVT was not studied [7]. In contrast, HUERTA et al. [8] found that there was little association between asthma diagnosis in primary health care and either DVT or PE. Thus, less severe asthma that is expected to occur in primary care does not seem to be an important risk factor for DVT or PE [8]. Divergence in the importance of asthma as a risk factor for VTE therefore exists.

DVT and PE are different manifestations of one disease, namely VTE, depending on whether the thrombus results in an embolus or not [9]. However, studies suggest that pulmonary embolism and DVT may not always have the same risk factors, as exemplified by the factor $\mathrm{V}$ paradox [9]. Another example that PE and DVT may not always have the same risk factors is that heart disease (myocardial infarction or heart failure) was found to be a stronger risk factor for PE than for DVT in a Danish nationwide study by SøRENSEN et al. [10]. In a recent study by VAN LANGEVELDE et al. of 99 patients with PE, simultaneous DVT was found in only 40 patients (40\%) with total-body magnetic resonance imaging scans [11]. The authors stated that the possibility that $\mathrm{PE}$ arises de novo in the lungs due to local inflammation-driven coagulation needs to be considered [11]. However, the methodology used in the cited studies [9-11] does not allow for final conclusions on this topic.

We hypothesised that the risk for DVT and PE could be different in asthma patients. We therefore determined the risk for a first event of hospitalisation for PE, DVT or a combination of DVT and PE $(\mathrm{DVT}+\mathrm{PE})$ in patients hospitalised with asthma in a nationwide case-control study.

\section{Material and methods}

This study was approved by the Ethics Committee of Lund University, Sweden. The data sources used in the present case-control study of adult VTE patients between 1981 and 2010 were provided by Statistics Sweden and the National Board of Health and Welfare. To assess the risk of DVT and PE among patients hospitalised for asthma in Sweden, comprehensive registers and healthcare data from multiple nationwide sources were linked using the unique 10-digit personal identification (ID) numbers assigned at birth or immigration to all Swedish residents for life [12-16]. The personal ID numbers were replaced by a serial number in order to preserve confidentiality. The Swedish Total Population Register, which contains data on annual sociodemographic factors, provided information about sex, country of birth, mortality and birth year. The Swedish Hospital Discharge Register includes hospitalisations in Sweden from 1964 to 2010, and is nationwide with complete coverage (100\%) of all hospitalisations in Sweden from 1987. The coverage of the Swedish Hospital Discharge Register was $71 \%$ in 1982 and $86 \%$ in 1984. This register provides information about hospital diagnoses of asthma, DVT, PE and comorbidities. Diagnoses in the Swedish Hospital Discharge Register were identified by the International Classification of Disease (ICD) codes (versions ICD-8, ICD-9 and ICD-10) and date of admission (registration).

\section{Nationwide case-control study}

Individuals in the study needed to be 18 years or older and born in Sweden. We used all primary and secondary diagnosis positions with first date of VTE. We defined PE based on the following codes: ICD-8 (450), ICD-9 (415B) and ICD-10 (I26). For DVT we used ICD-8 (451), ICD-9 (451) and ICD-10 (I80). Individuals with only superficial thrombophlebitis (ICD-9 451A and ICD-10 I800) were excluded from the study. If a patient had several admissions for VTE on different dates, the date of the first admission for VTE was used. We refer to this admission date as the index date. Asthma disease diagnosis was defined by the ICD codes 493 (ICD-8 and ICD-9) and J45 and J46 (ICD-10) before the index date (i.e. the first date for PE, DVT or PE+DVT). Asthma patients with pulmonary differential diagnoses such as chronic obstructive bronchitis, bronchiectasis and interstitial lung diseases were excluded (supplementary table S1). Patients' educational level was categorised into four groups: low ( $0-9$ years), middle (10-11 years), high 
education $(\geqslant 12$ years), and a fourth category that included individuals with unknown education level. An individual could only be selected as a control individual once in each of the three analyses.

A case-control study was performed and the matching was without replacement between 1981 and 2010. The controls needed to be alive on the index date. The case and control groups were then used to select exacted matched controls. We excluded patients with DVT, PE or DVT+PE before 1981 in order to ensure that only cases with a first event of VTE were included. In total, 6854 DVT+PE cases, 76494 DVT cases and $114366 \mathrm{PE}$ cases were matched according to age, sex and education with five controls for each case in the respective study groups.

Adjustment was done in the multivariate models for the following comorbidities, all of which are potential risk factors for VTE (supplementary table S2 for ICD codes): atrial fibrillation/flutter, obesity, psychiatric disease, coronary heart disease (CHD), congestive heart failure, peripheral vascular disease, cerebrovascular disease, varicose veins and inflammatory bowel disease that were identified by such a diagnosis before the index date. Pregnancy or delivery, fractures/trauma, or surgery within 90 days were also identified before the index date. To identify patients with cancer we used cancer diagnoses within 5 years before and 1 year after the index date.

\section{Ascertainment of diagnosis}

Data from the Swedish Patient Register diagnoses have high validity for a range of conditions, including asthma and VTE $[13,14,17,18]$. Overall, the positive predictive value of the inpatient register was identified in a recent review to be $85-95 \%$ for most diagnoses $[13,14]$. ÖRTQVIST et al. have examined the validity of diagnoses of asthma in a sample of asthma patients [18]. They found a positive predictive value of 0.75-0.94 depending on age. The validity for cardiovascular disorders such as VTE, stroke and myocardial infarction is approximately $90-95 \%[13,14,17]$.

\section{Statistical analysis}

We used asthma as the main predictor variable in the models. We tabulated the frequency and proportion of $\mathrm{PE}, \mathrm{DVT}$ and PE+DVT cases and population controls for the main and confounder variables. In order to study the effects of asthma on the response variables (PE, DVT and PE+DVT) we used conditional logistic regression. Odds ratios were computed with $95 \%$ confidence intervals. Univariate (crude) and multivariate (adjusted) odds ratios were calculated. Estimates were adjusted for potential confounding (i.e. comorbidities) in the multivariate conditional logistic regression models. An interaction term was introduced to test for potential effect modification on a multiplicative scale. We calculated stratified odds ratios according to time of asthma diagnosis before index date, using the classifications 0-90 days before, 91-365 days before, 1-5 years before and $>5$ years before. We also performed stratified analysis by age, with groupings of $18-50$ years, $51-75$ years and $\geqslant 76$ years old.

\section{Results}

The characteristics and distribution of cases and matched (1:5) population controls are presented in table 1 . Among PE patients, $8.8 \%$ were affected by PE before 51 years of age, in comparison to $15.8 \%$ of patients in the DVT group and $16.1 \%$ in the PE+DVT group. There were $49.9 \%$ women in the PE+DVT group, $54.6 \%$ in the PE group and $56.4 \%$ in the DVT group. The most common comorbidities in all three groups (PE, DVT, PE+DVT) were cancer, surgery, CHD, cerebrovascular disease and psychiatric disease.

The odds ratios for PE, DVT and PE+DVT in asthma patients compared to those without asthma are presented in table 2. In Model 1 (crude model), patients with asthma compared to those without asthma had an odds ratio of 1.60 for PE, 1.66 for DVT and 1.73 for PE+DVT. Adjusting for other comorbidities in Model 2 reduced the estimates slightly. The adjusted odds ratio for PE in asthma patients compared with those without asthma was 1.43 (95\% CI 1.37-1.50), for DVT was 1.57 (95\% CI 1.49-1.66) and for PE+DVT was 1.62 (95\% CI 1.34-1.96).

We examined statistical interactions on a multiplicative scale between age and asthma (results not shown) in the three case-control studies in order to detect possible effect modifications. There were significant statistical interactions on a multiplicative scale between asthma and age in the PE and DVT groups, but we did not find a statistical interaction in the PE+DVT group. The odds ratio for PE decreased from about 2.18 to 1.54 in older patients (table 3). The odds ratio for DVT decreased from about 2.01 before the age of 50 years to 1.55 after the age of 75 years (table 3 ). We also examined the association between time since asthma diagnosis and PE, DVT and PE+DVT (table 4). The odds ratios were highest if asthma was diagnosed within 3 months before PE, DVT or PE+DVT. The unadjusted odds ratios for PE decreased from 2.2 to 1.3; for DVT, the odds ratios decreased from 2.2 to 1.5. However, the odds ratios did not decreased to any major degree for PE+DVT (table 4). 
TABLE 1 Characteristics and distribution of cases and matched population controls (1:5)

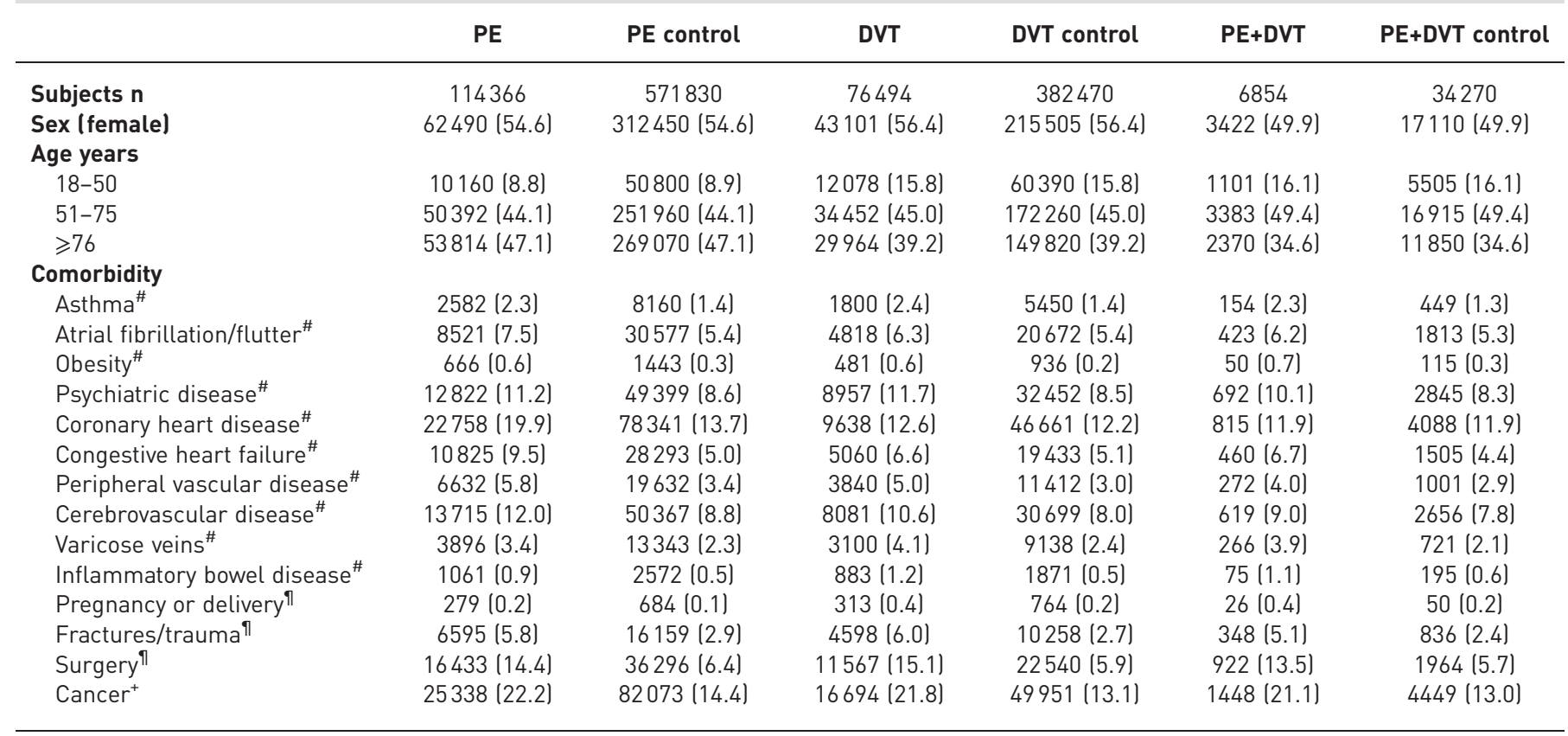

Data are presented as $\mathrm{n}(\%)$. PE: pulmonary embolism; DVT: deep vein thrombosis; PE+DVT: combined pulmonary embolism and deep venous thrombosis. ${ }^{\#}$ : any time before index date; ${ }^{\text {I }}$ : within 90 days before index date; ${ }^{+}$: 5 years before index date and 1 year after diagnosis.

Table 5 shows descriptive statistics for individuals who had asthma before the index date among cases and controls. Asthma was most common among women. The most common age group for asthma was age 51-75 years. Almost 50\% of asthma patients had just one hospital admission with asthma before the first event of PE, DVT or PE+DVT.

TABLE 2 Association between asthma and pulmonary embolism, deep venous thrombosis, and combined pulmonary embolism and deep venous thrombosis

\begin{tabular}{lcc} 
Asthma as a risk factor for & Model 1 $^{\#}$ & Model 2 $^{\text {q }}$ \\
\hline PE & $1.60(1.53-1.67)$ & $1.43(1.37-1.50)$ \\
DVT & $1.66(1.58-1.76)$ & $1.57(1.49-1.66)$ \\
PE+DVT & $1.73(1.44-2.08)$ & $1.62(1.34-1.96)$
\end{tabular}

Data are presented as OR $(95 \% \mathrm{Cl}) . \mathrm{PE}$ : pulmonary embolism; DVT: deep vein thrombosis; PE+DVT: combined pulmonary embolism and deep venous thrombosis. "\#: univariate model; " : multivariate model adjusted for atrial fibrillation/flutter, obesity, psychiatric disease, coronary heart disease, congestive heart failure, peripheral vascular disease, cerebrovascular disease, varicose veins, inflammatory bowel disease, pregnancy or delivery, fractures/trauma, surgery, cancer.

TABLE 3 Age-stratified odds ratio for pulmonary embolism and deep venous thrombosis

\begin{tabular}{llll}
$\begin{array}{l}\text { Asthma as a risk } \\
\text { factor for }\end{array}$ & Age 18-50 years & Age 51-75 years & Age $^{\Uparrow} \mathbf{7 6}$ years $^{+}$ \\
\hline PE & $2.18(1.86-2.55)$ & $1.58(1.47-1.69)$ & $1.54(1.44-1.64)$ \\
DVT & $2.01(1.71-2.35)$ & $1.72(1.58-1.86)$ & $1.55(1.43-1.68)$
\end{tabular}

Data are presented as OR (95\% CI). PE: pulmonary embolism; DVT: deep vein thrombosis. ${ }^{\text {: }}$ PE case $n=10$ 160 and control $n=50800$; DVT case $n=12078$ and control $n=60390$; ${ }^{\circ}$ : PE case $n=50392$ and control $n=251$ 960; DVT case $n=34452$ and control $n=172260 ;{ }^{+}$: PE case $n=53814$ and control $n=269070$; DVT case $n=29$ 964 and control $n=149820$. 
TABLE 4 Odds ratio of asthma as a risk factor for pulmonary embolism, deep venous thrombosis and combined pulmonary embolism and deep venous thrombosis according to time of asthma diagnosis before index date

\begin{tabular}{|c|c|c|c|c|c|}
\hline & & \multicolumn{4}{|c|}{ Time of asthma diagnosis before index date } \\
\hline & & 3 months & 3-12 months & $1-5$ years & $>5$ years \\
\hline \multirow[t]{2}{*}{ PE } & Unadjusted & $2.24(2.06-2.45)$ & $1.57(1.40-1.75)$ & $1.52(1.40-1.65)$ & $1.27(1.17-1.38)$ \\
\hline & Adjusted $^{\#}$ & $1.77(1.62-1.94)$ & $1.40(1.25-1.56)$ & $1.38(1.26-1.50)$ & $1.23(1.13-1.34)$ \\
\hline \multirow[t]{2}{*}{ DVT } & Unadjusted & $2.20(1.96-2.47)$ & $1.66(1.45-1.90)$ & $1.55(1.40-1.71)$ & $1.49(1.36-1.63)$ \\
\hline & Adjusted $^{\#}$ & $1.79(1.59-2.02)$ & $1.60(1.38-1.81)$ & $1.48(1.33-1.64)$ & $1.48(1.35-1.63)$ \\
\hline \multirow[t]{2}{*}{ PE+DVT } & Unadjusted & $2.21(1.48-3.31)$ & $1.54(0.93-2.55)$ & $1.44(1.01-2.04)$ & $1.82(1.34-2.45)$ \\
\hline & Adjusted $^{\#}$ & $2.00(1.33-3.04)$ & $1.34(0.80-2.25)$ & $1.32(0.92-1.89)$ & $1.79(1.32-2.43)$ \\
\hline
\end{tabular}

Data are presented as OR $(95 \%$ CI). PE: pulmonary embolism; DVT: deep vein thrombosis; PE+DVT: combined pulmonary embolism and deep venous thrombosis. \#: adjusted for atrial fibrillation/flutter, obesity, psychiatric disease, coronary heart disease, congestive heart failure, peripheral vascular disease, cerebrovascular disease, varicose varices, inflammatory bowel disease, pregnancy or delivery, fractures/ trauma, surgery, cancer.

\section{Relationship between the number of hospital admissions for asthma and risk of $P E, D V T$ and $P E+D V T$}

The associations between the average number of hospital admissions for asthma and PE, DVT and $\mathrm{PE}+\mathrm{DVT}$ are presented in supplementary table S3. The crude and adjusted odds ratios increased with an increased number of hospital admissions for asthma. The pattern was similar for PE, DVT and PE+DVT. For instance, compared to the non-asthmatic controls, the adjusted odds ratio for PE increased with the number of hospital admissions for asthma, from 1.34 (95\% CI 1.26-1.41) for those having one to two asthma admissions, up to 1.82 (95\% CI 1.64-2.02) for those having more than four asthma admissions. For those with three to four asthma admissions the adjusted odds ratio was 1.42 (95\% CI 1.27-1.60).

\section{Discussion}

The present study shows that asthma is a risk factor for PE, DVT and DVT+PE. It confirms the previous two studies by MAJOOR et al. and CHUNG et al. showing that asthma increases the risk for PE [6, 7]. However, our large-scale study extends previous findings because we show that asthma is also associated with the first

TABLE 5 Characteristics of asthma, cases and population controls

\begin{tabular}{|c|c|c|c|c|c|c|}
\hline & PE & Population controls & DVT & Population controls & PE+DVT & Population controls \\
\hline Sex (female) & $1677(65.0)$ & 4958 (60.8) & 1166 (64.8) & $3412(62.6)$ & $91(59.1)$ & $261(58.1)$ \\
\hline $18-50$ & $359(13.9)$ & $959(11.8)$ & $319(17.7)$ & 886 (16.3) & 27 (17.5) & 85 (18.9) \\
\hline \multicolumn{7}{|l|}{$\begin{array}{l}\text { Number of registrations } \\
\text { (for asthma) }\end{array}$} \\
\hline 1 & $1193(46.2)$ & 4149 (50.9) & $842(46.8)$ & $2874(52.7)$ & $73(47.4)$ & $231(51.5)$ \\
\hline 2 & $449(17.4)$ & $1476(18.1)$ & $313(17.4)$ & $985(18.1)$ & 30 (19.5) & 83 (18.5) \\
\hline 3 & $243(9.4)$ & 791 (9.7) & $175(9.7)$ & 502 (9.2) & $16(10.4)$ & 48 (10.7) \\
\hline $4-10$ & $481(18.6)$ & $1290(15.8)$ & $306(17.0)$ & $808(14.8)$ & 28 (18.2) & 60 (13.4) \\
\hline Unknown & $547(21.2)$ & $1923(23.6)$ & 238 (13.2) & 858 (15.7) & 19 (12.3) & $46(10.2)$ \\
\hline
\end{tabular}

Data are presented as $n(\%)$, unless otherwise stated. PE: pulmonary embolism; DVT: deep vein thrombosis; PE+DVT: combined pulmonary embolism and deep venous thrombosis. 
event of isolated DVT and combined PE and DVT. We also found a time-dependent effect with the highest risk of VTE within 3 months of asthma admission. However, the odds ratio for VTE was increased in asthma patients during the whole follow-up time. The less pronounced impact of asthma on PE risk in our study compared to the Dutch study by MAJOOR et al. could be due to a larger study size with inclusion of asthma cases from the whole Swedish population [6]. The Dutch study included only asthma patients from three Dutch tertiary asthma clinics, suggesting the inclusion of more severe cases. Our results also confirm the findings from the study by CHung et al. that showed that asthma increases PE risk [7]. In the Taiwanese study by CHung et al. [7], the asthma patients were younger than in the present study, which could have contributed to the higher PE risk associated with asthma found by CHUNG et al. compared to the present study. In our study, the odds ratio was highest in the youngest asthma patients. In the study by CHung et al., the hazard ratio increased with an increased number of emergency room visits and hospital admissions [7]. Our results confirmed this finding by CHUNG et al., with a higher odds ratio with increased numbers of hospital admissions for asthma. Thus, asthma severity seems to be of importance. A low asthma severity could explain why the study by HUERTA et al. found little association between asthma diagnosis in primary health care and either DVT or PE [8]. Asthma cases treated in primary care are most likely less severe than hospital-treated cases. Thus, asthma severity could also explain the higher risk found by MAJOor et al. among asthma patients from three Dutch tertiary asthma clinics [6]. Asthma patients treated in tertiary asthma clinics are likely to reflect the most severe cases [6]. There are also many important ethnic differences both for asthma and VTE $[19,20]$ that could contribute to the lower odds ratios observed in our Swedish study compared to the study from Taiwan by CHung et al. [7].

The mechanism of the association between asthma and VTE is not completely clear. Several causes of the increased risk of VTE among asthma patients may be of importance. Asthma is associated with inflammation [4], and inflammation is associated with thrombosis [1-3]. Inflammation is also expected to be stronger in more severe cases, which could explain this observation. An increased hypercoagulability with enhanced thrombin formation and impaired fibrinolysis have been observed among asthma patients [21]. Whether an increased hypercoagulable response in younger asthma patients could explain the higher odds ratios in younger individuals remains to be determined. Glucocorticoids that are often used to treat asthma are associated with endothelial dysfunction, hypercoagulability and stasis [22-24]. Glucocorticoids have also been shown to decrease fibrinolysis, as suggested by VAN ZAANE et al. and MAJOOR et al. [25, 26]. Systemic glucocorticoids are more likely to be used in severe asthma than in less severe asthma, which also could contribute to the association between asthma severity and VTE risk.

\section{Strength and limitations}

Our study strengths include the large number of cases and controls and the nationwide population-based coverage with complete hospital history for all cases and controls in a uniform tax-supported healthcare system. We were also able to confirm that there are more PE cases in older ages and that DVT is relatively more common at younger ages [27]. All data were collected prospectively, thereby limiting information bias from differential misclassification. Still, the accuracy of our findings depends on the quality of coding of VTE, asthma and comorbidities $[13,14,17,18]$. Registrations of asthma in hospital registries are accurate, but they are incomplete measures of asthma. We had limited data on the asthma disease severity although we used number of hospitalisations for asthma as an indicator of severity. Differences in severity of asthma could explain the relatively lower odds ratios found in our study compared with previous published studies. Mild asthma treated only by primary care providers would not be recorded. Thus, these individuals could be included in the control group, lowering the odds ratio. However, because we included only hospitalised cases, we only included those with the most severe asthma and less severe asthma is most likely associated with a lower risk of VTE than more severe hospitalised asthma. A further limitation is that the combined DVT and PE is likely to reflect cases with both symptomatic DVT and PE because asymptomatic PE occurs frequently among DVT cases and asymptomatic DVT occurs frequently in PE cases [28-30]. These cases are usually not recognised in the clinic. However, symptomatic PE or DVT are more likely to be severe [30], i.e. occlusive, suggesting that the symptomatic cases with combined PE and DVT included in the present study are relatively severe. Finally, we could not exclude residual and unmeasured confounding although, in an attempt to do so, we adjusted our analyses for several comorbidities.

In conclusion, asthma was associated with an increased risk of PE, DVT and PE+DVT to a similar degree. Thus, the inflammation conferred by asthma seems to have systemic (and not just local) prothrombotic effects with increased risk of both DVT and PE. These conclusions could guide the search for potential mechanisms behind the hypercoagulability in asthma patients. 


\section{References}

1 Ramagopalan SV, Wotton CJ, Handel AE, et al. Risk of venous thromboembolism in people admitted to hospital with selected immune-mediated diseases: record-linkage study. BMC Med 2011; 9: 1-8.

2 Zöller B, Li X, Sundquist J, et al. Risk of pulmonary embolism in patients with autoimmune disorders: a nationwide follow-up study from Sweden. Lancet 2012; 379: 244-249.

3 Zöller B, Li X, Sundquist J, et al. Autoimmune diseases and venous thromboembolism: a review of the literature. Am J Cardiovasc Dis 2012; 2: 171-183.

Martinez FD, Vercelli D. Asthma. Lancet 2013; 382: 1360-1372.

de Boer JD, Majoor CJ, van 't Veer C, et al. Asthma and coagulation. Blood 2012; 119: 3236-3244.

Majoor CJ, Kamphuisen PW, Zwinderman AH, et al. Risk of deep vein thrombosis and pulmonary embolism in asthma. Eur Respir J 2013; 42: 655-661.

7 Chung WS, Lin CL, Ho FM, et al. Asthma increases pulmonary thromboembolism risk: a nationwide population cohort study. Eur Respir J 2014; 43: 801-807.

8 Huerta C, Johansson S, Wallander MA, et al. Risk factors and short-term mortality of venous thromboembolism diagnosed in the primary care setting in the United Kingdom. Arch Intern Med 2007; 167: 935-943.

9 van Langevelde K, Flinterman LE, van Hylckama Vlieg A, et al. Broadening the factor V Leiden paradox: pulmonary embolism and deep-vein thrombosis as 2 sides of the spectrum. Blood 2012; 120: 933-946.

10 Sørensen HT, Horvath-Puho E, Lash TL, et al. Heart disease may be a risk factor for pulmonary embolism without peripheral deep venous thrombosis. Circulation 2011; 124: 1435-1441.

11 van Langevelde K, Srámek A, Vincken PW, et al. Finding the origin of pulmonary emboli with a total-body magnetic resonance direct thrombus imaging technique. Haematologica 2013; 98: 309-315.

12 Rosen M, Hakulinen T. Use of disease registers. In: Ahrens W, Pigeot I, eds. Handbook of epidemiology. Berlin, Springer-Verlag, 2005; pp. 231-252.

13 Ludvigsson JF, Andersson E, Ekbom A, et al. External review and validation of the Swedish national inpatient register. BMC Public Health 2011; 11: 450.

14 Zöller B. Nationwide family studies of cardiovascular diseases - clinical and genetic implications of family history. EMJ Cardiology 2013; 1: 102-113.

15 Ludvigsson JF, Almqvist C, Bonamy AK, et al. Registers of the Swedish total population and their use in medical research. Eur J Epidemiol 2016; 31: 125-136.

16 Ludvigsson JF, Otterblad-Olausson P, Pettersson BU, et al. The Swedish personal identity number: possibilities and pitfalls in healthcare and medical research. Eur J Epidemiol 2009; 24: 659-667.

17 Rosengren A, Fredén M, Hansson P-O, et al. Psychosocial factors and venous thromboembolism: a long-term follow-up study of Swedish men. J Thromb Haemost 2008; 6: 558-564.

18 Örtqvist AK, Lundholm C, Wettermark B, et al. Validation of asthma and eczema in population-based Swedish drug and patient registers. Pharmacoepidemiol Drug Saf 2013; 22: 850-860.

19 Tang L, Hu Y. Ethnic diversity in the genetics of venous thromboembolism. Thromb Haemost 2015; 114: 901-909.

20 Igartua C, Myers RA, Mathias RA, et al. Ethnic-specific associations of rare and low-frequency DNA sequence variants with asthma. Nat Commun 2015; 6: 5965.

21 Bazan-Socha S, Mastalerz L, Cybulska A, et al. Asthma is associated with enhanced thrombin formation and impaired fibrinolysis. Clin Exp Allergy 2016; 46: 932-944.

22 Sneeboer MM, Majoor CJ, de Kievit A, et al. Prothrombotic state in patients with severe and prednisolone-dependent asthma. J Allergy Clin Immunol 2016; 137: 1727-1732.

23 Coelho MC, Santos CV, Vieira Neto L, et al. Adverse effects of glucocorticoids: coagulopathy. Eur J Endocrinol 2015; 173: M11-M21.

24 Miljic P, Miljic D, Cain JW, et al. Pathogenesis of vascular complications in Cushing's syndrome. Hormones (Athens) 2012; 11: 21-30.

25 van Zaane B, Nur E, Squizzato A, et al. Systematic review on the effect of glucocorticoid use on procoagulant, anti-coagulant and fibrinolytic factors. J Thromb Haemost 2010; 8: 2483-2493.

26 Majoor CJ, Sneeboer MM, de Kievit A, et al. The influence of corticosteroids on hemostasis in healthy subjects. J Thromb Haemost 2016; 14: 716-723.

27 Silverstein MD, Heit JA, Mohr DN, et al. Trends in the incidence of deep vein thrombosis and pulmonary embolism: a 25-year population-based study. Arch Intern Med 1998; 158: 585-593.

28 Ryu JH, Olson EJ, Pellikka PA. Clinical recognition of pulmonary embolism: problem of unrecognized and asymptomatic cases. Mayo Clin Proc 1998; 73: 873-879.

29 Huisman MV, Büller HR, ten Cate JW, et al. Unexpected high prevalence of silent pulmonary embolism in patients with deep venous thrombosis. Chest 1989; 95: 498-502.

30 Hirsh J, Hull RD, Raskob GE. Epidemiology and pathogenesis of venous thrombosis. J Am Coll Cardiol 1986; 8: 104B-113B. 